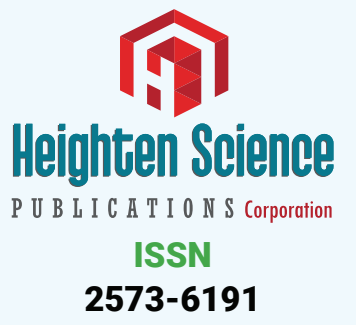

*Address for Correspondence: Rohit Kulshrestha Consulting Orthodontist, Private Practice Mumbai, India, Email: kulrohit@gmail.com

Submitted: 20 October 2017

Approved: 01 November 2017

Published: 02 November 2017

Copyright: @ 2017 Singh A, et al. This is an open access article distributed under the Creative Commons Attribution License, which permits unrestricted use, distribution, and reproduction in any medium, provided the original work is properly cited.

Keywords: Motivation; Oral hygiene practices; Oral hygiene awareness
Research Article

\section{Assessment of Oral Hygiene awareness in Geriatric patients attending OPD at ESIC Dental College, Rohini, New Delhi}

\author{
Abhishek Singh ${ }^{1}$, Aanchal Sahani ${ }^{2}$, Rohit Kulshrestha ${ }^{3 *}$, Sahil \\ Gupta ${ }^{4}$, Deepak Passi ${ }^{5}$ and Mayank Gupta ${ }^{6}$ \\ 'Senior Consultant, Yashoda Hospital, New Delhi, India \\ 2Intern, ESIC Dental College and Hospital, Rohini, New Delhi India \\ ${ }^{3}$ Consulting Orthodontist, Private Practice, Mumbai, India \\ ${ }^{4}$ Intern. ESIC Dental College and Hospital, Rohini, New Delhi, India \\ ${ }^{5}$ Senior Lecturer, Department of Oral Surgery, ESIC Dental College and Hospital, Rohini, New \\ Delhi, India \\ ${ }^{6}$ Senior Resident, GTB Hospital, New Delhi, India
}

\section{Abstract}

Aim: To assess and learn oral health awareness and hygiene practices among geriatric patients and also to identify important barriers in the establishment of oral health services, disease prevention and oral health promotion programmes for the same.

Materials and Methods: A total of 500 patients in the age group of 50 years and above were selected using random sampling technique. A self-administered structured questionnaire including 20 multiple choice questions was given to them. The results were analyzed using percentage.

Results: The result of this study shows an acute lack of oral hygiene awareness and limited knowledge of oral hygiene practices. In Rohini, few people use tooth brush.

Conclusions: Hence, there is an urgent need for comprehensive educational programs to promote good oral health and impart education about correct oral hygiene practices.

\section{Introduction}

Oral hygiene is the practice of keeping the mouth and teeth clean to prevent dental problems like dental caries, gingivitis, and bad breath. Oral health is important for appearances, sense of well-being and also for overall physical and mental health. Oral health can affect quality of life directly, and has been linked to sleeping problems, behavioural and developmental effects in children. Oral health for older people is a priority action area for the World Health Organisation Global Oral Health Programme [1]. Age-friendly primary oral health care, disease prevention and oral health promotion are of major concerns to WHO. The public health challenge related to oral health intervention for older people was emphasized by the 2007 World Health Assembly Resolution WHA60.17 on an action plan for oral health [2]. In 2007, WHO initiated a global survey to tend to the needs of older people to improve their oral health care. Good oral health practices are necessary from a young age to ensure positive long term dental health and hygiene. The oral health of children is very important towards their overall growth, development and well-being. 
Oral health is an integral part of general health; therefore, its disregarding will give rise the negative health and social consequences. Oral health status is often determined by the amount deposited on the surfaces of teeth and poor oral hygiene introduced as a predisposing factor to periodontal diseases and associated with cardiovascular diseases and even pre-term low-birth weight infants. In contrast, healthy oral behaviours reduce the amount of deposits particularly plaque on the surfaces of teeth. Toothbrush use is grossly limited in under developed areas. The toothpick is traditionally utilized for dental cleaning. Regular brushing of teeth after principal meals is not practiced universally [3]. Yazdani et al. [4], showed that all of 417 students had dental plaque, and $93 \%$ had gingival bleeding on at least one index tooth. Over the past two decades, increasing levels of tooth decay in children and adolescents has been observed in developing countries compared with developed countries. Taking care of one's teeth and gums is as important for senior citizens as it is for youngsters-if not more so. After all, the oral hygiene of an elderly person has a direct and significant bearing on quality of their life: how comfortably he or she can eat, speak, and smile.

Poor oral health can lead to tooth decay and periodontal (gum) disease. Untreated periodontal disease can worsen chronic health conditions such as heart disease, arthritis and diabetes. Maintaining oral health as we grow older presents its own special challenges and may be a difficult task. That's why it is often important for the grown children of older adults to take an active role in seeing to their parents needs in this crucial area. In the process of helping older persons with their oral care, we have the opportunity to see problems and changes in the mouth that may be important. Hence, the present study was carried to assess the oral health awareness among people of Rohini New Delhi, to learn about their oral hygiene practices and to provide insight into educational programs that have to be organized by health care professionals to assess oral hygiene awareness in geriatric patients.

\section{Materials and Methods}

The study sample consisted of 500 patients above 50 years of age attending dental OPD at Esic Dental College and Hospital, Rohini were selected. First, a pilot study was done on 10 patients present at OPD where they were asked to answer many questions relating to oral hygiene awareness. From this study, irrelevant questions that couldn't be correlated to any worthy conclusion were found and eliminated while the rest were kept for the main study. A self-made questionnaire was prepared consisting of 20 questions which aided to assess oral hygiene awareness. Out of the 20 questions 10 are related to knowledge, 5 are related to attitude and 5 are related to practice. With prior permission from the concerned authorities, the preformed questionnaire was handed over to the samples. They were provided explanation of the purpose of the study. The investigators were available throughout to answer any query regarding the questionnaire. After collection, the data was coded, computerized and analyzed.

\section{Results}

In the present study, questionnaire was distributed to 500 patients who were selected voluntarily. Of the 500 patients, $70 \%$ were male and $30 \%$ were female. Almost $82 \%$ of total sample used tooth brush as a method to clean their teeth (Figure 1). In response to other methods of cleaning their teeth, $70 \%$ uses toothpaste, $10 \%$ indicated use of neem stick and $20 \%$ use tooth powder with finger. $44 \%$ brushed their teeth twice a day, $33 \%$ of them were brushing once a day, $23 \%$ were brushing thrice a day. Around $75 \%$ of the subjects brush their teeth in horizontal direction, which is the most dangerous method of brushing (Figure 2).

$56 \%$ of the patients used inter-dental aid to clean the proximal areas in which $58 \%$ used inter-dental brush, $36 \%$ used toothpick which should not be used, and only $6 \%$ showed evidence of using dental floss (Figure 3). Only 12\% used mouth wash and 39\% rinsed with water post meal (Figure 4). 36\% of people noticed bleeding from gums especially during tooth brushing which is known as tooth brush trauma (Figure 5). 

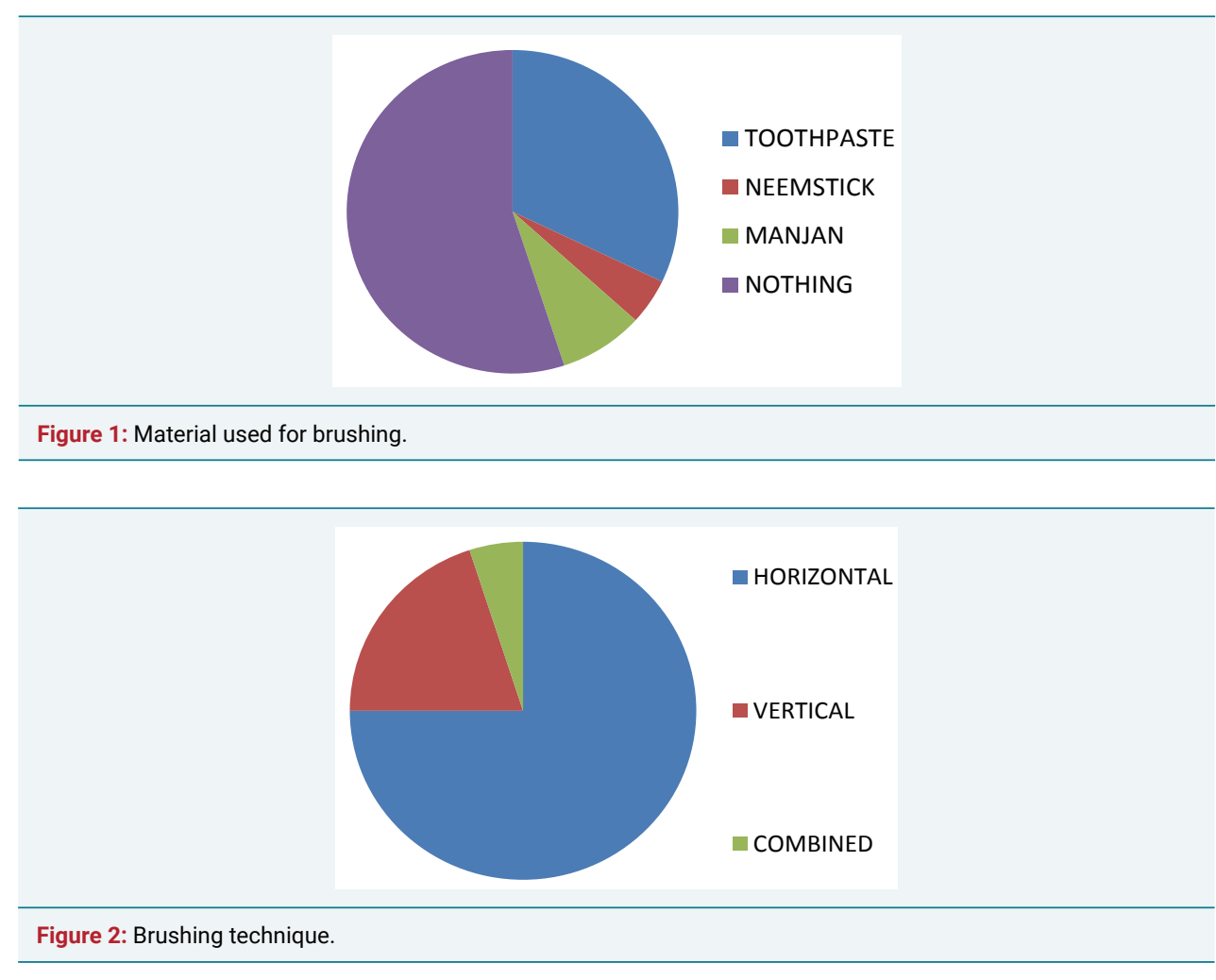

\begin{tabular}{ll}
\hline & $\begin{array}{l}\text { Interdental Brush } \\
\text { Eloss } \\
\text { wooth pick }\end{array}$ \\
\hline Figure 3: Use of inter-dental aid. \\
\hline
\end{tabular}

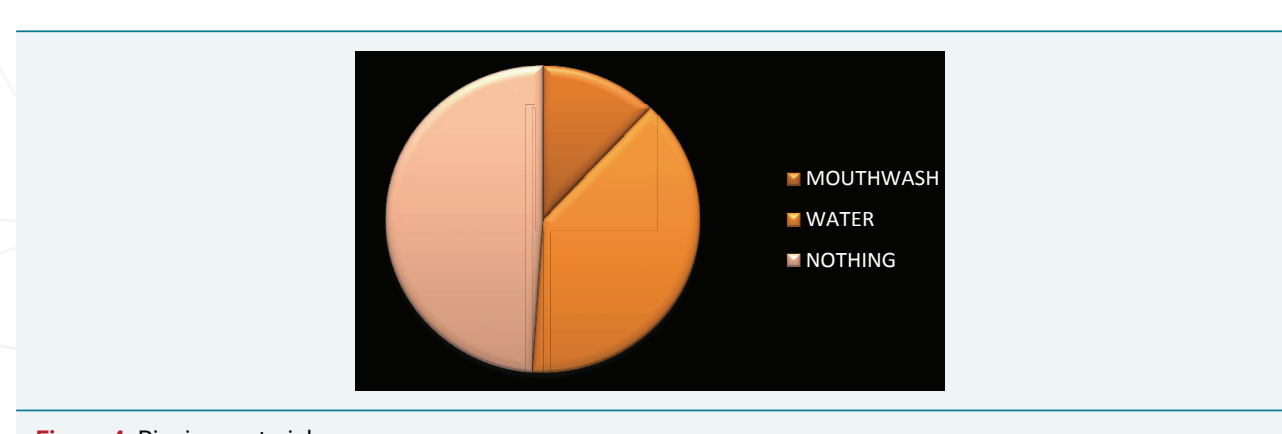

Figure 4: Rinsing material.

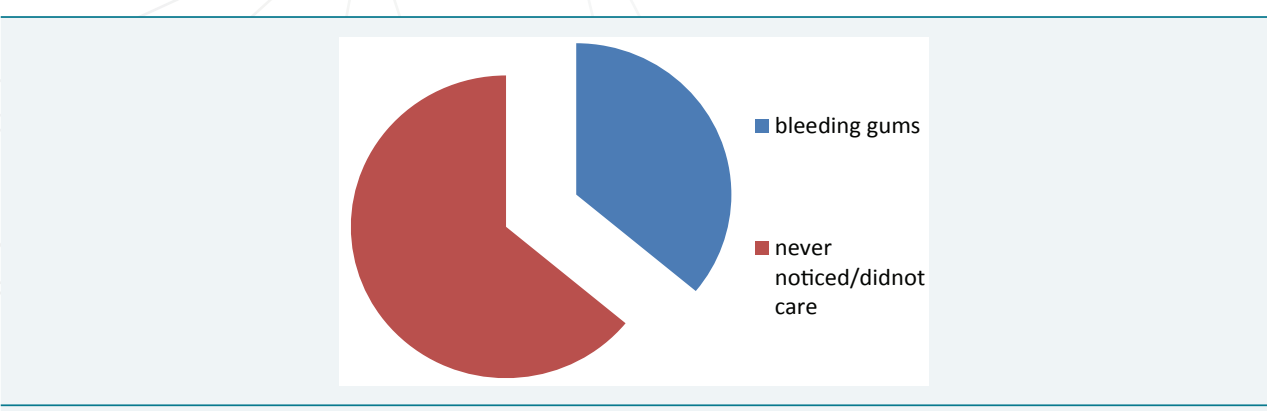

Figure 5: Bleeding gums. 
$46 \%$ of subjects experienced malodor and $54 \%$ are aware that there teeth look dirty but still only $40 \%$ have visited their dentist once/twice and surprisingly only $18 \%$ would want to visit for dental check-up in future.

\section{Questionnaire}

Name -

Age -

Gender -

Occupation -

Address -

Socio-economic status - Upper class Lower Class

Lower Middle Class Upper Middle Class

1. Do you brush your teeth? YES NO

2. If yes then how many times? 123

3. What do you brush your teeth with? Paste Neem Powder Salt

4. What do you use to brush your teeth? Brush Finger Else

5. If brush than what type of brush Soft Bristles Hard Bristles

6. What direction do you brush in? Straight Horizontal Vertical

7. Do you change your brush often? YES NO

8. If yes then in how much time? After 3 months 6 months 1 year

9. Do you use anything to clean between your teeth? YES NO

10. Do you use any of the following? Floss Toothpick Inter-dental Brush

11. Do you rinse after eating food? YES NO

12. If yes then with what? Water Mouth Wash

13. Have you ever had malodour? YES NO

14. Have you ever brushed very forcefully? YES NO

15. If yes then have you experienced bleeding from the gums? YES NO

16. Due to forceful brushing have you felt sensitivity? YES NO

17. Do you your teeth look dirty? YES NO

18. Have you ever visited the Dentist YES NO

19. Have you ever got your teeth cleaned? YES NO

20. Will you get your teeth cleaned in the future? YES NO

\section{Discussion}

It is a well-known fact in India that oral hygiene has less importance and has mostly remained as an ignored, unimportant and unrealized health problem. Most diseases show their first appearance through oral signs and symptoms and they remain undiagnosed or 
untreated because of this missing awareness. According to the consumer usage and attitudes survey which was done in 2005, the most shocking of revelations was that more than half of the Indian population did not use a tooth brush and only $51 \%$ brushed their teeth using a tooth brush and toothpaste [1]. Over the past 20 years significant measures have been made to promote oral hygiene and major emphasis has also been made on prevention of diseases rather than the treatment aspect of oral diseases. Healthy teeth can last us a lifetime with the proper preventive dental care. Preventive oral health knowledge, behaviour, and its practice are the important ways of keeping our teeth healthy. Hence, in this study attempts were made to evaluate preventive oral health knowledge, practice, and behaviour of the population of New Delhi. Although brushing was the commonly used method of cleaning, the percentage of subjects brushing their teeth twice daily is $44 \%$ which is quite good as compared with $23 \%$ of the older patients at Vyas Dental College and Hospital, Jodhpur but very less as compared to $75 \%$ of the elderly [5]. It is noteworthy that $75 \%$ of the respondents brushed their teeth using traditional horizontal method, which will jeopardize the tooth structure. This finding is in agreement with that of the study done by Zhu et al. [6], where $60 \%$ of the sample did the same. Surprisingly, fifty-six percent change their toothbrush once in 3 months, and $18 \%$ change their brush only when it is useless. There is generally a failure in the use of dental floss as a preventive tool. Only $6 \%$ of the subjects has used dental floss, which is similar to a study conducted by Jamjoom in Saudi Arabia in 2001 [7]. In contrast, Hamilton and Couby [8] found that a high percentage (44\%) of the sample they studied in north eastern Ontario used dental floss. Reason for this may be the significant resource allocation to health education programs that are carried out in Canada. This emphasizes the urgent need for educating and motivating the public to use this efficient method for oral health care. Only $39 \%$ of the sample population rinses their mouth after eating food. This missing and very basic method of maintaining oral hygiene is a clear indication of lack of awareness.

$12 \%$ of subjects used a mouthwash. Interestingly enough, they used it to treat malodour. Furthermore, 48\% reported halitosis. This study is in contrast with that of an epidemiologic survey of the general population of Japan where $24 \%$ of the individuals examined complained about bad breath [9].36\% percent of the total subjects reported bleeding gums. This study is in agreement with studies of Ashley et al. [10], and Buhlin et al. [11], who showed that self-reported bleeding gums was high in percentage. This study is in contrast with the studies of Nagarajan [12], and Pushpanjali in India. Kallio et al. [13], who showed that most of the patients did not notice bleeding from gums. Our study showed that $44 \%$ of the subjects visited a dentist when they were in pain/need, which is similar to the study done by Al-Shammari [14], in 2007 , where $54.5 \%$ of the participants reported visiting a dentist only when they have pain. Unsurprisingly, standards of oral health are very poor in India, with a large proportion of the population being affected due to poor socio-economic conditions. In addition to this, two thirds of people have never seen a dentist [15-17]. Missing awareness about the crucial role of regular dental checkups in preventing and detecting dental diseases is another gap in public education $[18,19]$. As dentists, it is our responsibility to educate and motivate people to visit a dentist.

\section{Conclusion}

The indifferent results of this study show that oral hygiene awareness is very poor in Rohini area of New Delhi as same seen in various parts of India. The information on developments in vital combination of oral hygiene, oral diagnosis, and overall health needs to be spread by us, the dentists. Establishing and demonstrating a connection between good oral hygiene and its relation directly to overall health is the need of the hour. To achieve this critical goal this process will have to be taken at all levels in society starting from a definite beginning with our patients. We, as dentists, will have to keep reinforcing the importance of correcting all aspects related with brushing and flossing along with the importance of regular checkups. The task of spreading this awareness extends beyond our private clinical practices and general masses should 
be targeted to have a large scale effect. This can be achieved by various outreach programs and relevant public health awareness measures through various mediums, such as media, newspapers, Internet, and organizing social activities and events for creating awareness. All of these and more innovative methods of reaching the public and this will not only ensure a healthy individual but a healthy over all society as well.

\section{References}

1. Dilip CL. Health status, treatment requirements, knowledge and attitude towards oral health of police recruits in Karnataka. J Indian Assoc Public Health Dent. 2005; 5: 20-34. Ref.: https://goo.gl/GQ1nVW

2. Jiang $H$, Petersen PE, Peng B, Tai B, Bian Z. Self-assessed dental health, oral health practices, and general health behaviors in Chinese urban adolescents. Acta Odontol Scand. 2005; 63: 343-52. Ref.: https://goo.gl/rtpXzK

3. Gilbert AD, Nuttal NM. Self-reporting of periodontal health status. Br Dent J. 1999; 186: 241-244. Ref.: https://goo.gl/CuUV5x

4. Yazdani R, Vehkalahti MM, Nouri M, Murtomaa H. School-based education to improve oral cleanliness and gingival health in adolescents in Tehran, Iran. Int J Paediatr Dent. 2009.19; 4: 274-281. Ref.: https://goo.gl/fJqbQw

5. Jain N, Mitra D, Ashok KP, Dundappa J, Soni S, et al. Oral hygiene-awareness and practice among patients attending OPD at Vyas Dental College and Hospital, Jodhpur. J Indian Soc Periodontol. 2012; 16: 524-528. Ref.: https://goo.gl/BcMvWY

6. Zhu L, Petersen PE, Wang HY, Bian JY, Zhang BX. Oral health knowledge, attitudes and behaviour of adults in China. Int Dent J. 2005; 55: 231-241. Ref.: https://goo.gl/P5fNhw

7. Jamjoom HM. Preventive Oral Health Knowledge and Practice in Jeddah, Saudi Arabia. J KAU: Med Sci. 2001; 9: 17-25. Ref.: https://goo.gl/ASCtBV

8. Hamilton ME, Coulby WM. Oral health knowledge and habits of senior elementary school students. $J$ Publ Health Dent. 1991; 51: 212-218. Ref.: https://goo.gl/pzZzBq

9. Miyazaki H, Sakao S, Katoh Y, Takehara T. Oral malodor in the general population of Japan. Bad Breath: Research Perspectives. Israel: Ramot Publishing: Tel Aviv University. 1995; 119-136. Ref.: https://goo.gl/f7AG3P

10. Ashley FP. Role of dental health education in preventive dentistry. In prevention of dental disease. 3rd ed. In: Murray JJ, editor. Oxford: Oxford University Press. 1996; 406-414.

11. Buhlin K, Gustaffon A, Anderson K, Hakansson K, Klinge B. Validity and limitations of self reported periodontal health. Community Dent Oral Epidemiol. 2002; 30: 431-437. Ref.: https://goo.gl/s8NUsV

12. Nagarajan S, Pushpanjali K. Self-assessed and clinically diagnosed periodontal health status among patients visiting the outpatient department of a dental school in Bangalore, India. Indian J Dent Res. 2008; 19: 243-246. Ref.: https://goo.gl/M1njbB

13. Kallio P, Nordblad A, Croucher R, Ainamo J. Self-reported gingivitis and bleeding gums among adolescents in Helsinki. Community Dent Oral Epidemiol. 1994; 22: 277-282. Ref.: https://goo.gl/rwvXWV

14. Al-Shammari KF, Al-Ansari JM, Al-Khabbaz AK, Dashti A, Honkala EJ. Self-reported oral hygiene habits and oral health problems of Kuwaiti adults. Med Princ Pract. 2007; 16: 15-21. Ref.: https://goo.gl/SnynfW

15. Freeman R, Maizels J, Wyllie M, Sheiham A. The relationship between health-related knowledge, attitudes and dental health behaviors in 14-16-year-old adolescents. Community Dent Health. 1993; 10: 397-404. Ref.: https://goo.gl/MpFWCq

16. Kay EJ, Locker D. A systematic review of the effectiveness of health promotion aimed at improving oral health. Community Dent Oral Epidemiol. 1998; 26: 132-144. Ref.: https://goo.gl/ZWknqC

17. Woodgroove J, Cumberbatch G, Gylbier S. Understanding dental attendance behaviour. Community Dent Health. 1987; 4: 215-221. Ref.: https://goo.gl/L8XuwY

18. Tervonen $\mathrm{T}$, Knuttila M. Awareness of dental disorders and discrepancy between objective and subjective dental treatment needs. Community Dent Oral Epidemiol. 1988; 34: 345-348. Ref.: https://goo.gl/cdZcjW

19. Al-Beiruti N. Oral health behaviour among a sample of schoolteachers, physicians and Nurses in the Syrian Arab Republic. East. Mediterr Health J. 1997; 3: 258-262. Ref.: https://goo.gl/JR3dWj 